\title{
Retrospective Analyses of Cancer Trials or Existing Clinical Databases: Potential for Highly Clinically Relevant Hypothesis Generation
}

\author{
Maurie Markman \\ Cancer Treatment Centers of America, Eastern Regional Medical Center, Philadelphia, Pa., USA
}

The superiority of prospective clinical trial data in the quality of evidence provided to permit a meaningful conclusion is well recognized. In addition to the inherent difficulty of being certain all relevant information has been captured in a retrospective review of trials or previously existing databases when there was no previous intent to obtain the specific data in question, there is always the concern that patients inadvertently or intentionally may not have been included in the analysis or reasons for the decision to employ (or not employ) particular management strategies may not have been clearly discussed or even mentioned in the medical records.

Assuming a prospective analysis has been optimally considered and undertaken, the decision regarding what is relevant information to collect and review in a given study will have been decided prior to the initiation of the research. Further, at least in theory, such data will be obtained on all patients, and if not available an appropriate explanation for its absence should be provided.

However, despite these serious limitations, it is relevant to acknowledge the potential for retrospective studies to serve a critical role in hypothesis generation or as data to support or refute existing clinical paradigms or explanations for observed outcomes.

For example, 'conventional wisdom' appeared to declare that administering cisplatin directly into the perito- neal cavity as treatment of small volume residual advanced ovarian cancer could only realistically favorably impact patients with microscopic or very limited volume macroscopic cancer (following an attempt at primary maximal surgical cytoreduction) [1]. This conclusion resulted from the observation that the extent of direct tissue penetration of antineoplastic agents (such as cisplatin) following regional delivery was limited to a maximum of only a few millimeters from the surface.

However, in a retrospective review of a landmark phase 3 randomized trial that compared intraperitoneal to intravenous cisplatin in this clinical setting it was revealed that regional drug delivery to patients who initiated treatment with a single tumor mass of between 0.5 and $1.5 \mathrm{~cm}$ in maximal diameter achieved at least as much relative benefit from this strategy (when compared to systemic administration) as women who had only microscopic residual cancer [1,2]. (Note: This outcome may be hypothesized to result from the fact patients are treated with multiple drug instillations over several months, rather than a single treatment, with each treatment reducing the total volume of cancer to be treated with subsequent treatments.) Thus, this analysis challenged the existing 'conventional' thinking in this area, prompting investigators to explore therapy in the setting of larger volume cancer.

\section{KARGER}

Fax +4161306 1234 E-Mail karger@karger.ch www.karger.com
(C) 2012 S. Karger AG, Basel

$0030-2414 / 12 / 0836-0329 \$ 38.00 / 0$

Accessible online at:

www.karger.com/ocl
Maurie Markman, MD

Cancer Treatment Centers of America, Eastern Regional Medical Center

1331 East Wyoming Avenue

Philadelphia, PA 19124 (USA)

Tel. +1 215537 7502, E-Mail maurie.markman@ctca-hope.com 
In this issue of Oncology, two papers describe provocative retrospective analyses of existing databases [3, 4]. In one report, while examining an extensive database consisting of 430 patients with hepatocellular carcinomas diagnosed through the individual's participation in a wellorganized screening program, investigators noted somewhat surprising and quite provocative features associated with screened tumors that were $\leq 3$ versus $>3 \mathrm{~cm}$ in diameter at diagnosis [3]. Specifically, as a group, patients with the smaller sized tumors were found to have lower platelet counts and higher serum bilirubin levels at presentation.

While only hypothesis generating, this observation raises the fascinating and realistic possibility that the process of biological progression of hepatocellular cancers that grow in size relatively quickly may be different from that of the malignancies whose primary volume changes at a much slower pace. Clearly, this is a hypothesis worthy of retrospective exploration in other large institutional databases and in preclinical model systems.
Further, this report serves as an excellent example, similar to the previously noted experience of intraperitoneal cisplatin delivery in ovarian cancer, of a retrospective analysis that can challenge current thinking and provide insight into our understanding of quite complex biological phenomena.

\section{References}

1 Markman M: Critical thinking: an essential role in both the conduct and interpretation of gynecologic cancer clinical research. Gynecol Oncol 2008;108:462-465.

-2 Alberts DS, Liu PY, Hannigan EV, et al: Intraperitoneal cisplatin plus intravenous cyclophosphamide versus intravenous cisplatin plus intravenous cyclophosphamide for stage III ovarian cancer. N Engl J Med 1996;335:1950-1955.

3 Carr BI, Guerra V, De Giorgio M, Fagiuoli S, Pancoska P: Small hepatocellular carcinomas and thrombocytopenia. Oncology 2012;83:331338.

-4 Carr BI, Guerra V, Pancoska P: Thrombocytopenia in relation to tumor size in patients with hepatocellular carcinoma. Oncology 2012;83:339345. 